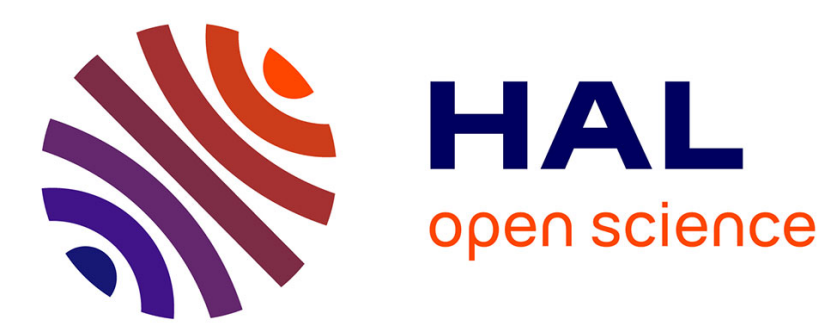

\title{
Self-oscillations of a vocal apparatus: a port-Hamiltonian formulation
}

Thomas Hélie, Fabrice Silva

\section{To cite this version:}

Thomas Hélie, Fabrice Silva. Self-oscillations of a vocal apparatus: a port-Hamiltonian formulation. Frank Nielsen and Frédéric Barbaresco. Geometric Science of Information: Third International Conference, GSI 2017, Paris, France, November 7-9, 2017, Proceedings, Springer International Publishing, pp.375-383, 2017, 3rd conference on Geometric Science of Information (GSI), 978-3-319-68445-1. hal01567464

\section{HAL Id: hal-01567464 \\ https://hal.science/hal-01567464}

Submitted on 23 Jul 2017

HAL is a multi-disciplinary open access archive for the deposit and dissemination of scientific research documents, whether they are published or not. The documents may come from teaching and research institutions in France or abroad, or from public or private research centers.
L'archive ouverte pluridisciplinaire HAL, est destinée au dépôt et à la diffusion de documents scientifiques de niveau recherche, publiés ou non, émanant des établissements d'enseignement et de recherche français ou étrangers, des laboratoires publics ou privés. 


\title{
Self-oscillations of a vocal apparatus: a port-Hamiltonian formulation
}

\author{
Thomas Hélie ${ }^{1}$ and Fabrice Silva ${ }^{2}$ \\ 1 S3AM Team, UMR STMS 9912, IRCAM-CNRS-UPMC, Paris, France \\ 2 Aix Marseille Univ., CNRS, Centrale Marseille, LMA, Marseille, France
}

\begin{abstract}
Port Hamiltonian systems (PHS) are open passive systems that fulfil a power balance: they correspond to dynamical systems composed of energy-storing elements, energy-dissipating elements and external ports, endowed with a geometric structure (called Dirac structure) that encodes conservative interconnections. This paper presents a minimal PHS model of the full vocal apparatus. Elementary components are: (a) an ideal subglottal pressure supply, (b) a glottal flow in a mobile channel, (c) vocal-folds, (d) an acoustic resonator reduced to a single mode. Particular attention is paid to the energetic consistency of each component, to passivity and to the conservative interconnection. Simulations are presented. They show the ability of the model to produce a variety of regimes, including self-sustained oscillations. Typical healthy or pathological configuration laryngeal configurations are explored.
\end{abstract}

\section{Motivations}

Many physics-based models of the human vocal apparatus were proposed to help understanding the phonation and its pathologies, with a compromise between the complexity introduced in the modelling and the vocal features that can be reproduced by analytical or numerical calculations. Except recent works based on finite elements methods applied to the glottal flow dynamics, most of the models rely on the description of the aerodynamics provided by van den Berg [1] for a glottal flow in static geometries, i.e., that ignores the motion of the vocal folds. Even if enhancements appeared accounting for various effects, they failed to represent correctly the energy exchanges between the flow and the surface of the vocal folds that bounds the glottis.

The port-Hamiltonian approach offers a framework for the modelling, analysis and control of complex system with emphasis on passivity and power balance [2]. A PHS for the classical body-cover model has been recently proposed [3] without connection to a glottal flow nor to a vocal tract, so that no self-oscillations can be produced. The current paper proposes a minimal PHS model of the full vocal apparatus. This power-balanced numerical tool enables the investigation of the various regimes that can be produced by time-domain simulations. Sec. 2 is a reminder on the port-Hamiltonian systems, Sec. 3 is dedicated to the description of the elementary components of the full vocal apparatus and their interconnection. Sec. 4 presents simulation and numerical results for typical healthy and pathological laryngeal configurations. 


\section{Port-Hamiltonian Systems}

Port-Hamiltonian systems are open passive systems that fulfil a power balance [2,4]. A large class of such finite dimensional systems with input $\mathbf{u}(t) \in$ $\mathbb{U}=\mathbb{R}^{P}$, output $\mathbf{y}(t) \in \mathbb{Y}=\mathbb{U}$, can be described by a differential algebraic equation

$$
\left(\begin{array}{c}
\dot{\mathbf{x}} \\
\mathbf{w} \\
-\mathbf{y}
\end{array}\right)=S(\mathbf{x}, \mathbf{w})\left(\begin{array}{c}
\nabla_{\mathbf{x}} H \\
\mathbf{z}(\mathbf{w}) \\
\mathbf{u}
\end{array}\right) \text {, with } S=-S^{T}=\left(\begin{array}{ccc}
\mathbf{J}_{\mathbf{x}} & -\mathbf{K} & \mathbf{G}_{\mathbf{x}} \\
\mathbf{K}^{T} & \mathbf{J}_{\mathbf{w}} & \mathbf{G}_{\mathbf{w}} \\
-\mathbf{G}_{\mathbf{x}}^{T} & -\mathbf{G}_{\mathbf{w}}^{T} & \mathbf{J}_{\mathbf{y}}
\end{array}\right)
$$

where state $\mathbf{x}(t) \in \mathbb{X}=\mathbb{R}^{N}$ is associated with energy $E=\mathcal{H}(\mathbf{x}) \geq 0$ and where variables $\mathbf{w}(t) \in \mathbb{W}=\mathbb{R}^{Q}$ are associated with dissipative constitutive laws $\mathbf{z}$ such that $P_{\text {dis }}=\mathbf{z}(\mathbf{w})^{T} \mathbf{w} \geq 0$ stands for a dissipated power. Such a system naturally fulfils the power balance $\mathrm{d} E / \mathrm{d} t+P_{\text {dis }}-P_{\text {ext }}=0$, where the external power is $P_{\text {ext }}=\mathbf{y}^{T} \mathbf{u}$. This is a straightforward consequence of the skew-symmetry of matrix $S$, which encodes this geometric structure (Dirac structure, see [2]). Indeed, rewriting Eq. (1) as $B=S A$, it follows that $A^{T} B=A^{T} S A=0$, that is,

$$
\nabla_{\mathbf{x}} \mathcal{H}(x)^{T} \dot{\mathbf{x}}+\mathbf{z}(\mathbf{w})^{T} \mathbf{w}-\mathbf{u}^{T} \mathbf{y}=0
$$

Moreover, connecting several PHS through external ports yields a PHS. This modularity is used in practice, by working on elementary components, separately.

\section{Vocal apparatus}

Benefiting from this modularity, the full vocal apparatus is built as the interconnection of the following elementary components: a subglottal pressure supply, two vocal folds, a glottal flow, and an acoustic resonator (see Fig. 1).

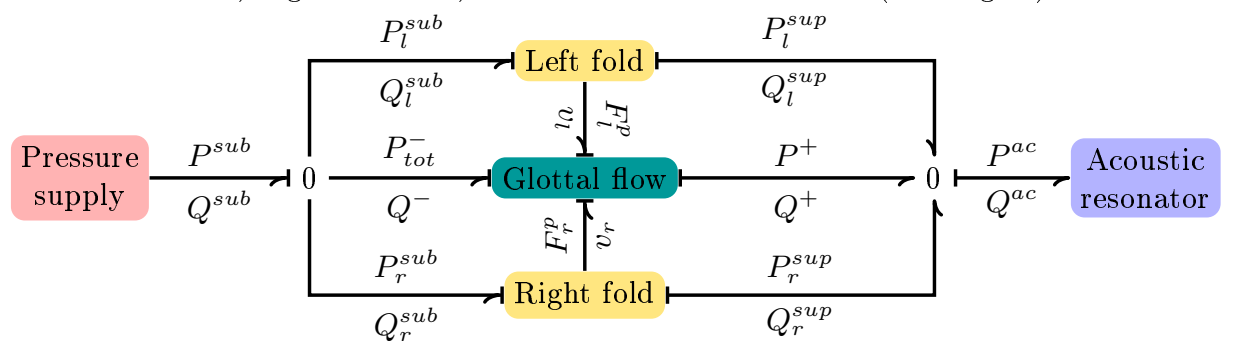

Fig. 1. Components of the vocal apparatus. The interconnection takes place via pairs of effort $(P)$ and flux $(Q)$ variables. The 0 connection expresses the equality of efforts and the division of flux. See Ref. [4] for an introduction to bond graphs.

\subsection{The one-mass model of vocal folds}

The left and right vocal folds $\left(\mathcal{F}_{i}=\mathcal{L}\right.$ or $\mathcal{R}$ with $i=l$ or $r$, respectively), are modelled as classical single-d.o.f. oscillators (as in Ref. [5], mass $m_{i}$, spring $k_{i}$ 
and damping $r_{i}$ ) with a purely elastic cover (as in Ref. [6], spring $\kappa_{i}$ ). Their dynamics relates the momentum $\pi_{i}$ of the mass, and the elongations $\xi_{i}$ and $\zeta_{i}$ of the body and cover springs, respectively, to the velocity $v_{i}=\dot{\zeta}_{i}+\dot{\xi}_{i}$ of the cover imposed by the glottal flow, and to the transverse resultants of the pressure forces on the upstream $\left(P_{i}^{s u b}\right)$ and downstream $\left(P_{i}^{s u p}\right)$ faces of the trapezoid-shaped structures (see Fig. 2, left part) :

$$
\dot{\pi}_{i}=-k_{i} \xi_{i}-r_{i} \dot{\xi}_{i}+\kappa_{i} \zeta_{i}-P_{i}^{s u b} S_{i}^{s u b}-P_{i}^{s u p} S_{i}^{s u p} .
$$

$F_{i}^{p}=-\kappa_{i} \zeta_{i}$ is the transverse feedback force opposed by the fold to the flow. The motion of the fold produces the additional flowrates $Q_{i}^{s u b}$ (pumping from the subglottal space, i.e., positive when the fold compresses) and $Q_{i}^{\text {sup }}$ (pulsated into the supraglottal cavity, i.e., positive when the fold inflates).

Port-Hamiltonian modelling of a vocal fold $\mathcal{F}_{i}$ :

$$
\begin{aligned}
& \mathbf{x}_{\mathcal{F}_{i}}=\left(\begin{array}{c}
\pi_{i} \\
\xi_{i} \\
\zeta_{i}
\end{array}\right), \mathbf{u}_{\mathcal{F}_{i}}=\left(\begin{array}{c}
P_{i}^{s u b} \\
P_{i}^{s u p} \\
v_{i}
\end{array}\right), \mathbf{y}_{\mathcal{F}_{i}}=\left(\begin{array}{c}
-Q_{i}^{s u b} \\
Q_{i}^{s u p} \\
-F_{i}^{p}
\end{array}\right), H_{\mathcal{F}_{i}}=\frac{1}{2} \mathbf{x}_{\mathcal{F}_{i}}^{T}\left(\begin{array}{cc}
1 / m_{i} & \\
& k_{i} \\
& \kappa_{i}
\end{array}\right) \mathbf{x}_{\mathcal{F}_{i}}, \\
& \mathbf{w}_{\mathcal{F}_{i}}=\dot{\xi}_{i}, \mathbf{z}_{\mathcal{F}_{i}}\left(\mathbf{w}_{\mathcal{F}_{i}}\right)=r_{i} \mathbf{w}_{\mathcal{F}_{i}}, \mathbf{J}_{\mathbf{w}}^{\mathcal{F}_{i}}=0, \mathbf{G}_{\mathbf{w}}^{\mathcal{F}_{i}}=\mathbb{O}_{1 \times 3}, \mathbf{J}_{\mathbf{y}}^{\mathcal{F}_{i}}=\mathbb{O}_{3 \times 3}, \\
& \mathbf{J}_{\mathbf{x}}^{\mathcal{F}_{i}}=\left(\begin{array}{ccc}
0 & -1 & 1 \\
1 & 0 & 0 \\
-1 & 0 & 0
\end{array}\right), \mathbf{K}^{\mathcal{F}_{i}}=\left(\begin{array}{l}
1 \\
0 \\
0
\end{array}\right), \text { and } \mathbf{G}_{\mathbf{x}}^{\mathcal{F}_{i}}=\left(\begin{array}{ccc}
-S_{i}^{s u b}-S_{i}^{\text {sup }} & 0 \\
0 & 0 & 0 \\
0 & 0 & 1
\end{array}\right) .
\end{aligned}
$$

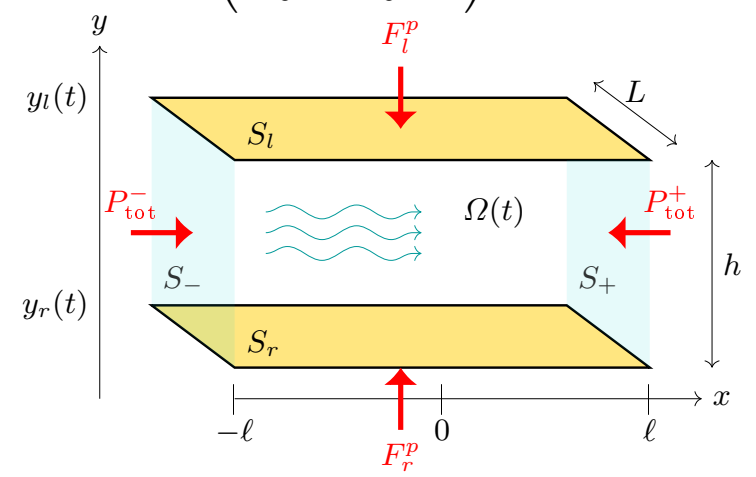

Fig. 2. Left: Schematic of a vocal fold. Right: Schematics of the glottal flow with open boundaries $S^{-}$and $S^{+}$and mobile walls $S_{l}$ and $S_{r}$.

\subsection{Glottal flow}

We consider a potential incompressible flow of an inviscid fluid of density $\rho$ between two parallel mobile walls located at $y=y_{l}(t)$ and $y=y_{r}(t)$, respectively. The glottis $\mathcal{G}$ has width $L$, length $2 \ell$ and height $h=y_{l}-y_{r}$, its mid-line being located at $y=y_{m}=\left(y_{r}+y_{l}\right) / 2$ (see Fig. 2, right part). The simplest kinematics for the fluid velocity $v(x, y)$ obeying the Euler equation

$$
\dot{v}+\frac{1}{\rho} \nabla\left(p+\frac{1}{2} \rho|v|^{2}\right)=0
$$


and satisfying the normal velocity continuity on the walls is given by:

$$
v=\left(\begin{array}{c}
v_{x} \\
v_{y}
\end{array}\right)=\left(\begin{array}{c}
v_{0}-x \frac{\dot{h}}{h} \\
\dot{y}_{m}+\frac{\dot{h}}{h}\left(y-y_{m}\right)
\end{array}\right) \quad \forall(x, y) \in \Omega=[-\ell, \ell] \times\left[y_{r}, y_{l}\right] .
$$

The velocity field is thus parametrised by four macroscopic quantities: $h$, its time derivative $\dot{h}$, and the mean axial and transverse velocities $v_{0}=\left\langle v_{x}>_{\Omega}\right.$ and $\dot{y}_{m}=<v_{y}>_{\Omega}$, respectively. Choosing these quantities as the state allows the exact reduction of the infinite-dimensional problem to a finite-dimension system. The pressure field $p(x, y, t)$ can also be obtained from Eq. (4), as well as the total pressure $p+\frac{1}{2} \rho|v|^{2}$, but are not expanded here for brevity.

The dynamics for the glottal flow is controlled by the mean total pressures $P_{\text {tot }}^{-}$and $P_{\text {tot }}^{+}$on the open boundaries $S^{-}(x=-\ell)$ and $S^{+}(x=+\ell)$, respectively, and the resultant $F_{r}^{p}$ and $F_{l}^{p}$ of the pressure forces on the right and left walls, respectively (see App. A for the derivation of the equations). The kinetic energy of the fluid on the domain writes as

$$
\varepsilon(t)=H_{\mathcal{G}}\left(\mathbf{x}_{\mathcal{G}}(t)\right)=\frac{1}{2}\left(m(h) v_{0}^{2}+m(h) \dot{y}_{m}^{2}+m_{3}(h) \dot{h}^{2}\right)
$$

with the total mass of the fluid $m(h)=2 \rho \ell L h(t)$, and the effective mass for the transverse expansion motion $m_{3}(h)=m(h)\left(1+4 \ell^{2} / h^{2}\right) / 12$. The energy could be written as a function of the momenta to yield a canonical Hamiltonian representation (see Ref. [7] for a similar PHS based on normalised momenta).

Downstream the glottis, the flow enters the supraglottal space which has a cross section area much larger than that of the glottis. For positive flowrate $\left(Q^{+}=\operatorname{Lhv}_{x}(\ell)>0\right)$, the flow separates from the walls at the end point of the (straight) channel. The downstream jet then spreads due to the shear-layer vortices until the jet has lost most of its kinetic energy into heat and fully mixed with the quiescent fluid. This phenomenon is modelled as a dissipative component with variable $\mathbf{w}_{\mathcal{G}}=Q^{+}$and dissipation function $\mathbf{z}_{\mathcal{G}}\left(\mathbf{w}_{\mathcal{G}}\right)=$ $(1 / 2) \rho\left(\mathbf{w}_{\mathcal{G}} / L h\right)^{2} \Theta\left(\mathbf{w}_{\mathcal{G}}\right)$ where $\Theta$ is the Heaviside step function. The pressure in the supraglottal space then writes $P^{+}=P_{t o t}^{+}-\mathbf{z}_{\mathcal{G}}$.

Port-Hamiltonian modelling of the glottal flow $\mathcal{G}$ :

$$
\begin{gathered}
\mathbf{x}_{\mathcal{G}}=\left(\begin{array}{c}
v_{0} \\
\dot{y}_{m} \\
\dot{h} \\
h
\end{array}\right), \mathbf{u}_{\mathcal{G}}=\left(\begin{array}{c}
P_{t o t}^{-} \\
P^{+} \\
F_{l}^{p} \\
F_{r}^{p}
\end{array}\right), \mathbf{y}_{\mathcal{G}}=\left(\begin{array}{c}
-Q^{-}=-L h v_{x}(-\ell) \\
+Q^{+}=L h v_{x}(\ell) \\
-v_{l}=+\dot{y}_{l} \\
-v_{r}=-\dot{y}_{r}
\end{array}\right), \\
H_{\mathcal{G}}\left(\mathbf{x}_{\mathcal{G}}\right)=\frac{m(h)}{2}\left(v_{0}^{2}+\dot{y}_{m}^{2}\right)+\frac{m_{3}(h)}{2} \dot{h}^{2} \\
\mathbf{w}_{\mathcal{G}}=Q^{+}, \mathbf{z}_{\mathcal{G}}=\frac{\rho}{2}\left(\frac{\mathbf{w}_{\mathcal{G}}}{L h}\right)^{2} \Theta\left(\mathbf{w}_{\mathcal{G}}\right), \\
\mathbf{J}_{\mathbf{w}}^{\mathcal{G}}=\mathbb{O}_{1 \times 1}, \mathbf{G}_{\mathbf{w}}^{\mathcal{G}}=\mathbb{O}_{1 \times 4} \text { and } \mathbf{J}_{\mathbf{y}}^{\mathcal{G}}=\mathbb{O}_{4 \times 4},
\end{gathered}
$$




$$
\mathbf{J}_{\mathbf{x}}^{\mathcal{G}}=\left(\begin{array}{cccc}
0 & 0 & 0 & 0 \\
0 & 0 & 0 & 0 \\
0 & 0 & 0 & -\frac{1}{m_{3}} \\
0 & 0 & \frac{1}{m_{3}} & 0
\end{array}\right), \mathbf{K}^{\mathcal{G}}=\left(\begin{array}{c}
\frac{L h}{m} \\
0 \\
-\frac{L \ell}{m_{3}} \\
0
\end{array}\right), \text { and } \mathbf{G}_{\mathbf{x}}^{\mathcal{G}}=\left(\begin{array}{cccc}
\frac{L h}{m} & -\frac{L h}{m} & 0 & 0 \\
0 & 0 & -\frac{1}{m^{2}} & \frac{1}{m_{1}} \\
\frac{L \ell}{m_{3}} & \frac{L \ell}{m_{3}} & -\frac{1}{2 m_{3}} & -\frac{1}{2 m_{3}} \\
0 & 0 & 0 & 0
\end{array}\right)
$$

\subsection{Vocal tract}

We assume a modal representation of the input impedance of the vocal tract as seen from the supraglottal cavity, i.e., the supraglottal pressure $P^{a c}$ is defined as the sum of pressure components $p_{n}$ (for $n=1, N$, denoted $P_{n}$ in the Fourier domain) related to the input flowrate $Q^{a c}$ through 2 nd order transfer functions:

$$
Z_{i n}(\omega)=\frac{P^{a c}(\omega)}{Q^{a c}(\omega)}=\sum_{n=1}^{N} \frac{P_{n}(\omega)}{Q^{a c}(\omega)}=\sum_{n=1}^{N} \frac{j \omega a_{n}}{\omega_{n}^{2}+j q_{n} \omega_{n} \omega-\omega^{2}}
$$

where $\omega$ is the angular frequency, $\omega_{n}$ are the modal angular frequencies, $q_{n}$ are the modal dampings and $a_{n}$ the modal coefficients. Each mode corresponds to a resonance of the vocal tract, and so to an expected formant in the spectrum of the radiated sound. We follow the convention defined in Ref. [8] for the internal variables of this subsystem.

Port-Hamiltonian modelling of the acoustic resonator $\mathcal{A}:$

$$
\begin{gathered}
\mathbf{x}_{\mathcal{A}}=\left(p_{1} / a_{1}, \ldots, p_{N} / a_{N}, \int_{0}^{t} p_{1}\left(t^{\prime}\right) d t^{\prime}, \ldots, \int_{0}^{t} p_{N}\left(t^{\prime}\right) d t^{\prime}\right)^{T}, \\
H_{\mathcal{A}}\left(\mathbf{x}_{\mathcal{A}}\right)=\sum_{n=1}^{N} \frac{1}{2}\left(\frac{p_{n}^{2}}{a_{n}}+\frac{\omega_{n}^{2}}{a_{n}}\left(\int_{0}^{t} p_{n}\left(t^{\prime}\right) d t^{\prime}\right)^{2}\right), \\
\mathbf{w}_{\mathcal{A}}=\left(p_{1}, \ldots, p_{N}\right)^{T}, \mathbf{z}_{\mathcal{A}}=\left(\frac{q_{1} \omega_{1}}{a_{1}} \mathbf{w}_{\mathcal{A} 1}, \ldots, \frac{q_{N} \omega_{N}}{a_{N}} \mathbf{w}_{\mathcal{A}, N}\right), \\
\mathbf{u}_{\mathcal{A}}=\left(Q^{a c}\right), \mathbf{y}_{\mathcal{A}}=\left(-P^{a c}\right), \\
\mathbf{J}_{\mathbf{x}}^{\mathcal{A}}=\left(\begin{array}{c}
\mathbb{O}_{N \times N}-\mathbb{I}_{N \times N} \\
\mathbb{I}_{N \times N}
\end{array} \mathbb{O}_{N \times N}\right), \mathbf{K}^{\mathcal{A}}=\left(\begin{array}{c}
\mathbb{I}_{N \times N} \\
\mathbb{O}_{N \times N}
\end{array}\right), \mathbf{G}_{\mathbf{x}}^{\mathcal{A}}=\left(\begin{array}{c}
1_{N} \\
\mathbb{O}_{N \times 1}
\end{array}\right), \\
\mathbf{G}_{\mathbf{w}}^{\mathcal{A}}=\mathbb{O}_{N \times 1}, \mathbf{J}_{\mathbf{w}}^{\mathcal{A}}=\mathbb{O}_{N \times N}, \text { and } \mathbf{J}_{\mathbf{y}}^{\mathcal{A}}=\mathbb{O}_{1 \times 1} .
\end{gathered}
$$

where $\mathbb{I}_{N \times N}$ is the identity matrix of $\operatorname{dim} N \times N$, and $1_{N}$ is the column vector $N \times 1$ filled with 1 .

\subsection{Full system}

We assume that the lower airways acts as a source able to impose the pressure $P^{s u b}$ in the subglottal space of the larynx. The flowrate $Q^{s u b}$ coming from this source splits into the flowrate $Q^{-}$entering the glottis and the flowrate $Q_{l}^{\text {sub }}$ and $Q_{r}^{s u b}$ pumped by the lower conus elasticus of the left and right vocal folds, respectively, so that $Q^{s u b}=Q^{-}+Q_{l}^{s u b}+Q_{r}^{s u b}$ with $P_{s u b}=P_{r}^{s u b}=P_{l}^{s u b}=P_{t o t}^{-}$. 
Conversely, the flowrate $Q^{+}$sums up with the flowrates $Q_{l}^{\text {sup }}$ and $Q_{r}^{\text {sup }}$ pulsated by the left and right vocal folds, respectively. The resulting flowrate $Q^{a c}$ that enters the acoustic resonator is then $Q^{a c}=Q^{+}+Q_{l}^{s u p}+Q_{r}^{\text {sup }}$ with $P^{a c}=P_{r}^{s u p}=$ $P_{l}^{\text {sup }}=P^{+}$.

The elementary components described above are now put together to assembly the full vocal apparatus. In order to simplify the Dirac structure, the ports of the subsystems have been chosen to be complementary: a port with sink convention is always connected to a port with source convention. As a result, it is trivial to expand the port Hamiltonian modelling of the full system with the following variables, dissipation functions, ports and energy:

$$
\begin{gathered}
\mathbf{x}=\left(\begin{array}{c}
\mathbf{x}_{\mathcal{R}} \\
\mathbf{x}_{\mathcal{L}} \\
\mathbf{x}_{\mathcal{G}} \\
\mathbf{x}_{\mathcal{A}}
\end{array}\right), \mathbf{w}=\left(\begin{array}{c}
\mathbf{w}_{\mathcal{R}} \\
\mathbf{w}_{\mathcal{L}} \\
\mathbf{w}_{\mathcal{G}} \\
\mathbf{w}_{\mathcal{A}}
\end{array}\right), \mathbf{z}=\left(\begin{array}{c}
\mathbf{z}_{\mathcal{R}} \\
\mathbf{z}_{\mathcal{L}} \\
\mathbf{z}_{\mathcal{G}} \\
\mathbf{z}_{\mathcal{A}}
\end{array}\right), \mathbf{u}=\left(P^{s u b}\right), \mathbf{y}=\left(-Q^{\text {sub }}\right), \\
H(\mathbf{x})=H_{\mathcal{R}}\left(\mathbf{x}_{\mathcal{R}}\right)+H_{\mathcal{L}}\left(\mathbf{x}_{\mathcal{L}}\right)+H_{\mathcal{G}}\left(\mathbf{x}_{\mathcal{G}}\right)+H_{\mathcal{A}}\left(\mathbf{x}_{\mathcal{A}}\right) .
\end{gathered}
$$

The matrices $\mathbf{J}_{\mathbf{x}}, \mathbf{K}, \mathbf{G}_{\mathbf{x}}, \mathbf{G}_{\mathbf{w}}, \mathbf{J}_{\mathbf{w}}$ and $\mathbf{J}_{\mathbf{y}}$ can be obtained using automated generation tools like the PyPHS software [9].

\section{Simulations and results}

We here briefly present some preliminary results. In the port-Hamiltonian modelling of the full system, the dissipation variables $\mathbf{w}$ do not explicitly depend on $\mathbf{z}$ (i.e., $\mathbf{J}_{w}=\mathbb{O}$ ), so that they can be eliminated leading to a differential realisation that can be numerical integrated (e.g., using the Runge-Kutta 4 scheme). The parameters have the following values: $m_{i}=0.2 \mathrm{~g}, r_{i}=0.05 \mathrm{~kg} / \mathrm{s}$, $L=11 \mathrm{~mm}, \ell=2 \mathrm{~mm}, \rho=1.3 \mathrm{~kg} / \mathrm{m}^{3}$. Due to the sparse data available on the input impedances of vocal tract notably in terms of modal amplitudes $a_{n}$, we consider a resonator with a single pole $(N=1)$ with $\omega_{n}=2 \pi \times 640 \mathrm{rad} / \mathrm{s}, q_{n}=.4$ and $a_{n}=1 \mathrm{M} \Omega$ (from Ref. [10]). The system is driven by a subglottal pressure $P^{s u b}$ that increases from 0 to $800 \mathrm{~Pa}$ within $20 \mathrm{~ms}$ and is then maintained.

In the first simulation, the folds are symmetric $\left(k_{r}=k_{l}=100 \mathrm{~N} / \mathrm{m}, \kappa_{r}=\right.$ $\left.\kappa_{l}=3 k_{r}\right)$ and initially separated by a width $h=1 \mathrm{~mm}$. In such conditions, the folds are pushed away from their rest position (until $h \sim 3 \mathrm{~mm}$ ), but this equilibrium does not become unstable and the system does not vibrate.

If some adduction is performed bringing the folds closer together $(h=0.1 \mathrm{~mm})$, the glottis first widens (until $h \sim 2 \mathrm{~mm}$ ) and the folds then start to vibrate and the acoustic pressure oscillates in the vocal tract (see Fig. 3, top). The sound is stable even if the two folds are slightly mistuned $\left(k_{r}=100 \mathrm{~N} / \mathrm{m}\right.$ and $\left.k_{l}=97 \mathrm{~N} / \mathrm{m}\right)$.

The right fold is then hardened $\left(k_{r}=150 \mathrm{~N} / \mathrm{m}\right)$. The system still succeeds to vibrate, but, as visible on Fig. 3 (bottom), the oscillation is supported by the soft left fold at first, and then this latter decays while the hardened right fold starts to vibrate and finally maintains the sound production (even if the oscillations seem intermittent). 

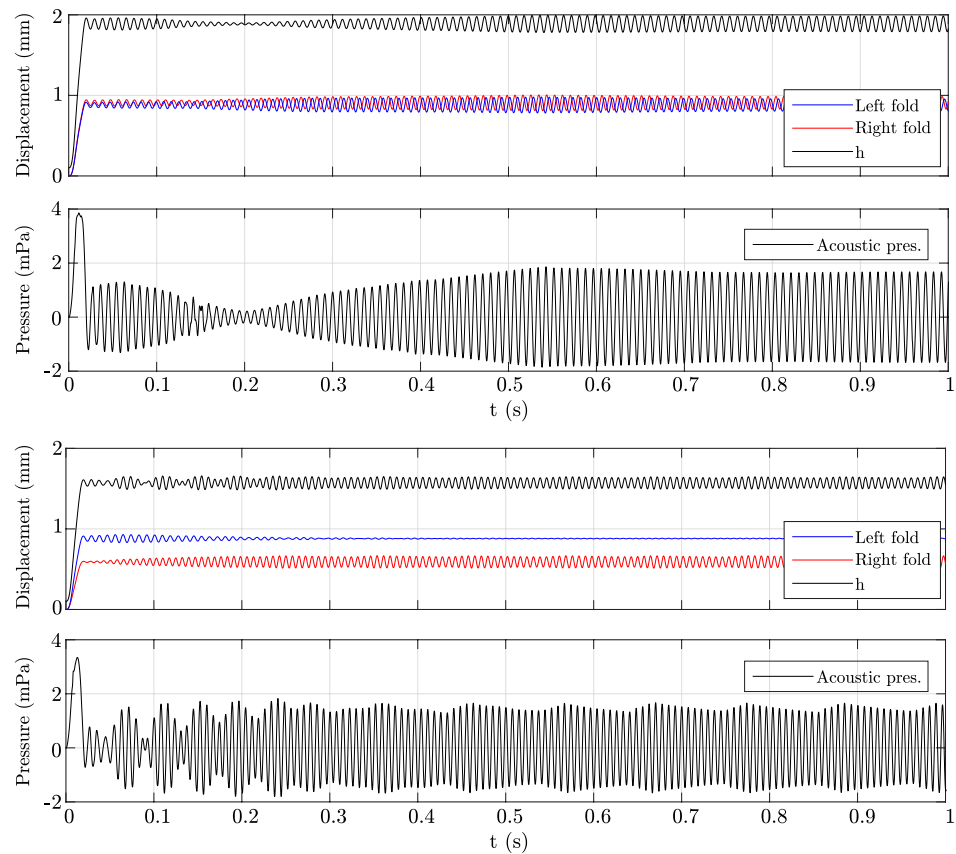

Fig. 3. Adducted (top) and asymmetric (bottom) configurations.

\section{Conclusion}

To the best knowledge of the authors, this paper proposes the first port-Hamiltonian model of a full vocal apparatus. This ensures passivity and the power balance. Simulations provide a variety of regimes that can be qualitatively related to aphonia (stable equilibrium), phonation (nearly periodic regimes) and dysphonia (irregular oscillations). This preliminary work provides a proof-of-concept for the relevance/interest of the passive and geometric approach.

Further work will be devoted to: (1) analyse regimes and bifurcations of the current model with respect to a few biomechanic parameters, (2) improve the realism of elementary components (separately), (3) account for possible contact between the vocal-folds, and (4) investigate on the synchronisation of coupled asymmetric vocals-folds and explore strategies to treat pathological voices [11].

Acknowledgement. The first author acknowledges the support of the Collaborative Research DFG and ANR project INFIDHEM ANR-16-CE92-0028.

\section{References}

1. van den Berg, J.: On the Air Resistance and the Bernoulli Effect of the Human Larynx. J. Acous. Soc. Am. 29(5), 626-631 (1957).

2. van der Schaft, A., Jeltsema, D.: Port-Hamiltonian Systems Theory: an Introductory Overview, Now Publishers Inc. (2014).

3. Encina, M., et al: Vocal fold modeling through the port-Hamiltonian systems approach. IEEE Conf. on Control App. (CCA), 1558-1563 (2015). 
4. Maschke, B., et al: An intrinsic Hamiltonian formulation of network dynamics: nonstandard Poisson structures and gyrators, J. Frankl. Inst. 329(5), 923-966 (1992).

5. Flanagan, J., Landgraf, L.: Self-Oscillating Source for Vocal-Tract Synthesizers. IEEE Trans. Audio Electroacous. AU-16(1), 57--64 (1968)

6. Awrejcewicz, J.: Numerical Analysis of the Oscillations of Human Vocal Cords. Nonlin. Dyn. 2, 35--52 (1991)

7. Lopes, N. , Hélie, T.: Energy balanced model of a jet interacting with a brass player's lip. Acta Acust united Ac. 102(1), 141-154 (2016).

8. Lopes, N.: Approche passive pour la modélisation, la simulation et l'étude d'un banc de test robotisé pour les instruments de type cuivre. PhD thesis, UPMC, Paris, 2016.

9. Falaize, A.: PyPHS: Passive modeling and simulation in python. Software available at https://afalaize.github.io/pyphs/ (last viewed on April 21st, 2017).

10. Badin, P., Fant, G.: Notes on vocal tract computation. STL-QPSR 25(2-3), 53-108 (1984).

11. Giovanni, A., et al: Nonlinear behavior of vocal fold vibration:The role of coupling between the vocal folds. J. Voice 13(4), 465-476 (1999)

\section{A Dynamics of the glottal flow}

The dynamics for the mean velocities can also be derived from the volume integration of the Euler equation (4). Using the gradient theorem, it comes that

$$
m(h) \dot{v}_{0}=\operatorname{Lh}(t)\left(P_{\text {tot }}^{-}-P_{\text {tot }}^{+}\right) \quad \text { and } \quad m(h) \ddot{y}_{m}=F_{r}^{p}-F_{l}^{p} .
$$

The energy balance for the glottal flow writes down as:

$$
\dot{\varepsilon}(t)+\int_{S^{-} \cup S^{+}}\left(p+\frac{1}{2} \rho|v|^{2}\right)(v \cdot n)+\int_{S_{l} \cup S_{r}} p(v \cdot n)=0
$$

where $n$ is the outgoing normal. As the normal velocity is uniform on the walls, the last term of the energy balance reduces to

$$
\int_{S_{l} \cup S_{r}} p(v \cdot n)=-\dot{y}_{r} \int_{S_{r}} p+\dot{y}_{l} \int_{S_{l}} p=\dot{y}_{m}\left(F_{l}^{p}-F_{r}^{p}\right)+\frac{\dot{h}}{2}\left(F_{r}^{p}+F_{l}^{p}\right) .
$$

The same applies on $S^{-} \cup S^{+}$where $v \cdot n= \pm v_{x}(x= \pm \ell)$ does not depend on $y$ :

$$
\begin{aligned}
\int_{S^{-} \cup S^{+}} p_{t o t}(v \cdot n) & =v_{x}(\ell) \int_{S^{+}} p_{t o t}-v_{x}(-\ell) \int_{S^{-}} p_{t o t} \\
& =L v_{0}\left(P_{t o t}^{+}-P_{t o t}^{-}\right)-L \ell \frac{\dot{h}}{h}\left(P_{t o t}^{+}+P_{t o t}^{-}\right) .
\end{aligned}
$$

Thus, $\dot{\varepsilon}=\dot{y}_{m}\left(F_{r}^{p}-F_{l}^{p}\right)-\frac{\dot{h}}{2}\left(F_{r}^{p}+F_{l}^{p}\right)+L h(t) v_{0}\left(P_{t o t}^{-}-P_{t o t}^{+}\right)+L \ell \dot{h}\left(P_{t o t}^{-}+P_{t o t}^{+}\right)$. In the meanwhile, the kinetic energy in Eq. (6) can be derived against time: $\dot{\varepsilon}=m(h)\left(v_{0} \dot{v}_{0}+\dot{y}_{m} \ddot{y}_{m}\right)+m_{3}(h) \dot{h} \ddot{h}+\frac{\partial H}{\partial h} \dot{h}$. The identification of the contribution of the mean axial and transverse velocities (see Eq. (8)) leads to the dynamics of the glottal channel expansion rate :

$$
m_{3} \ddot{h}=L \ell\left(P_{\text {tot }}^{-}+P_{\text {tot }}^{+}\right)-\frac{F_{r}^{p}+F_{l}^{p}}{2}-\frac{\partial H}{\partial h} .
$$

